\title{
Fault Analysis Study of IDEA
}

\author{
Christophe Clavier ${ }^{1}$, Benedikt Gierlichs ${ }^{2}$, and Ingrid Verbauwhede ${ }^{2}$ \\ 1 Gemalto, Security Labs \\ Avenue du Jujubier, ZI Athélia IV, 13705 La Ciotat Cedex, France \\ christophe.clavier@gemalto.com \\ ${ }^{2}$ K.U. Leuven, ESAT/SCD-COSIC \\ Kasteelpark Arenberg 10, B-3001 Leuven-Heverlee, Belgium \\ firstname.lastname@esat.kuleuven.be
}

\begin{abstract}
We present a study of several fault attacks against the block cipher IDEA. Such a study is particularly interesting because of the target cipher's specific property to employ operations on three different algebraic groups while not using substitution tables. We observe that the attacks perform very different in terms of efficiency. Although requiring a restrictive fault model, the first attack can not reveal a sufficient amount of key material to pose a real threat, while the second attack requires a large number of faults in the same model to achieve this goal. In the general random fault model, i.e. we assume that the fault has a random and a priori unknown effect on the target value, the third attack, which is the first Differential Fault Analysis of IDEA to the best of our knowledge, recovers 93 out of 128 key bits exploiting about only 10 faults. For this particular attack, we can also relax the assumption of cycle accurate fault injection to a certain extend.
\end{abstract}

Keywords: Collision Fault Analysis, Ineffective Fault Analysis, Differential Fault Analysis, IDEA, Random Fault Model

\section{Introduction}

The International Data Encryption Algorithm (IDEA) is a block cipher with an iterated round function, which encrypts 64-bit blocks of plaintext to 64-bit blocks of ciphertext using a 128-bit key. It was introduced by Xuejia Lai and James Massey in 1991 [9]. Today, this cipher is commonly available in cryptographic software packages such as PGP, SSH, and OpenSSL and is used in (embedded) cryptographic systems deployed by some operators of GSM networks and PayTV systems [13].

IDEA was designed to resist differential cryptanalysis $[3,9]$ in particular, but seems to be an algorithm difficult to cryptanalyze in general, even in reduced rounds versions. A part of IDEA's security is based on the 
fact that it applies operations on three different algebraic groups. Rather than comprising substitution tables, IDEA uses a multiplication operation to generate confusion. As a result, the progress in finding weaknesses in the cipher is relatively slow. The best known attack against IDEA at this time has been presented in 2007 by Biham, Dunkelman, and Keller [2]. It breaks 6 out of the 8.5 rounds of IDEA with $2^{64}-2^{52}$ pairs of plaintext/ciphertext and a time complexity equivalent to $2^{126.8}$ encryptions.

Since the algorithm is robust against classical cryptanalysis and widely deployed at the same time we think it is an interesting task to study its security under the threat of physical attacks. The fact that there exists only very little literature on this topic, e.g. [10], underlines the need for such a study.

In this work we do not explore all possible paths for physical attacks. While [10] presents some results for differential side channel analysis, we focus on fault analysis. We observe that Collision Fault Analysis (CFA) [7], which requires a restrictive fault model, does not allow to reveal a sufficient amount of key material to pose a real threat. Further, we show that Ineffective Fault Analysis (IFA) [5, 6], requiring the same fault model, allows to recover the entire key at the cost of a large number of fault injections. In this light, we propose a Differential Fault Analysis (DFA) [4] which seems to be of particular interest to an attacker, as it allows to retrieve a substantial part of the key from a small number of fault injections in the most general fault model. To the best of our knowledge, this is the first described Differential Fault Analysis of IDEA. The fact that we can also relax the assumption of cycle accurate fault injection to a certain extend renders this attack even more practical.

We describe IDEA in Section 2 and briefly recapitulate some fault analysis techniques in Section 3. The two following sections are respectively dedicated to the application of collision and Ineffective Fault Analysis to IDEA. Thereafter, we propose an efficient Differential Fault Analysis of this algorithm in Section 6 and provide results from extensive simulations of this attack in Section 7. We conclude our work in Section 8.

\section{IDEA}

IDEA consists of 8 identical rounds and an output transformation (reduced round). Each round is composed of XOR operations, additions modulo $2^{16}$ denoted by $\boxplus$, and multiplications modulo $\left(2^{16}+1\right)$ with 0 associated to $2^{16}$ denoted by $\odot$. Round $n(n=1, \ldots, 8)$ involves six 16 -bit subkeys $Z_{i}^{n}(i=1, \ldots, 6)$. The output transformation employs four additional 
subkeys $Z_{i}^{9}(i=1 \ldots, 4)$. Each subkey is composed of selected key bits according to IDEA's key schedule depicted in Figure 1.

\begin{tabular}{ccccccc}
\hline Round & $Z_{1}^{n}$ & $Z_{2}^{n}$ & $Z_{3}^{n}$ & $Z_{4}^{n}$ & $Z_{5}^{n}$ & $Z_{6}^{n}$ \\
\hline$n=1$ & $0-15$ & $16-31$ & $32-47$ & $48-63$ & $64-79$ & $80-95$ \\
$n=2$ & $96-111$ & $112-127$ & $25-40$ & $41-56$ & $57-72$ & $73-88$ \\
$n=3$ & $89-104$ & $105-120$ & $121-8$ & $9-24$ & $50-65$ & $66-81$ \\
$n=4$ & $82-97$ & $98-113$ & $114-1$ & $2-17$ & $18-33$ & $34-49$ \\
$n=5$ & $75-90$ & $91-106$ & $107-122$ & $123-10$ & $11-26$ & $27-42$ \\
$n=6$ & $43-58$ & $59-74$ & $100-115$ & $116-3$ & $4-19$ & $20-35$ \\
$n=7$ & $36-51$ & $52-67$ & $68-83$ & $84-99$ & $125-12$ & $13-28$ \\
$n=8$ & $29-44$ & $45-60$ & $61-76$ & $77-92$ & $93-108$ & $109-124$ \\
$n=9$ & $22-37$ & $38-53$ & $54-69$ & $70-85$ & & \\
\hline
\end{tabular}

Fig. 1. The IDEA key schedule

The input of each round $n(n=1, \ldots, 9)$ is split into four 16-bit words $X_{i}^{n}(i=1, \ldots, 4)$ which are processed by the IDEA round function according to Figure 2. The output transformation produces the ciphertext, denoted by $C_{i}(i=1, \ldots, 4)$.

The decryption and encryption functions are identical except for the subkeys involved. The decryption subkeys are derived from the encryption ones by considering them in reverse order and computing their respective inverses regarding the operations $\boxplus$ and $\odot$.

\section{Fault Analysis Techniques and Fault Models}

We now briefly recapitulate the three fault analysis techniques our attacks are based on and their respective fault models. The specific application of these techniques to the IDEA algorithm will be described in detail in the next sections. For all our attacks, we consider an unprotected software implementation of IDEA on a 16-bit platform, but the strategies can be easily adapted to 8-bit and 32-bit implementations. We assume that an adversary is able to induce at most one fault per encryption in a cycle accurate manner.

The first two types of fault analysis we consider both infer information about the key from the fact that two observed ciphertexts (resulting from one normal and one faulty execution) are identical. For these two analysis techniques we assume a fault model where an adversary is able to set the output of an arithmetic operation, $\boxplus$ or $\odot$ in the sequel, to a fixed and known (or guessable) value $a$. The particular value of this constant does not influence the attack and is usually assumed to be equal to zero. 


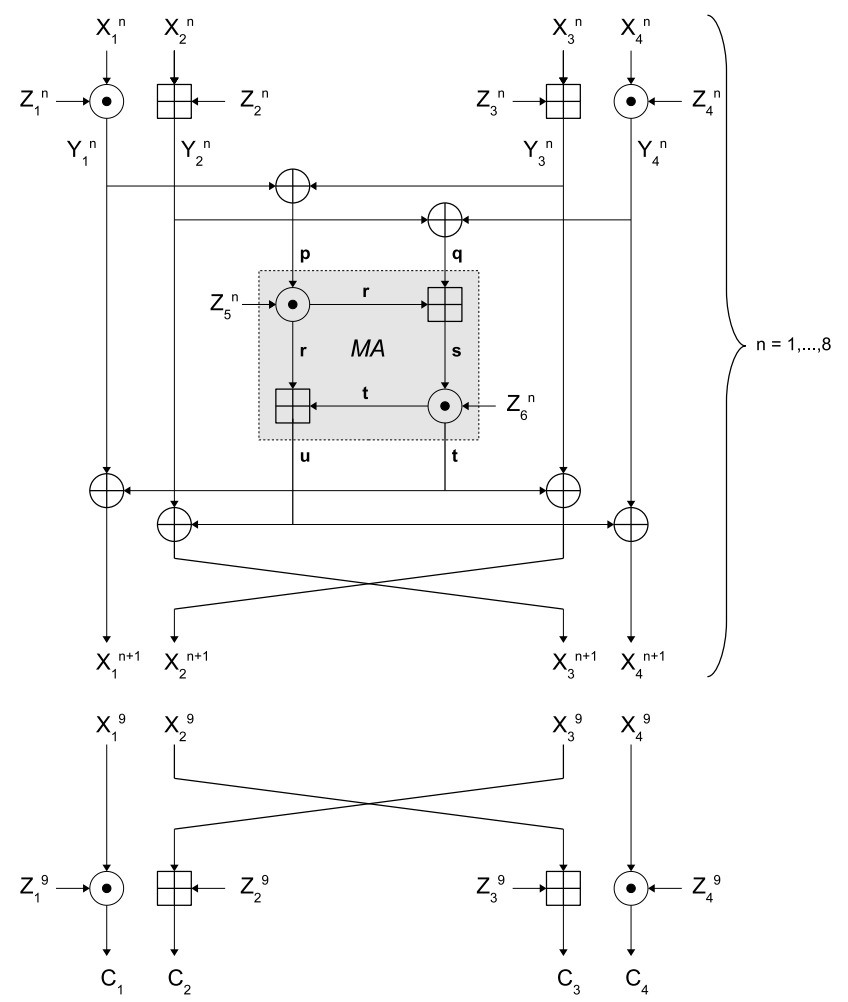

Fig. 2. IDEA round function and output transformation

Applying the first method, named Collision Fault Analysis (CFA) [1, 7], the adversary first obtains a faulty ciphertext corresponding to some arbitrary plaintext. Then she exhaustively searches for another input whose encryption eventually collides with this output. CFA is so a chosen message fault analysis which is particularly efficient since a single fault injection is usually sufficient to reveal some piece of information about the key.

Nevertheless, it is not always possible to obtain a collision with a faulted ciphertext by searching a message that provokes it. This is particularly the case if the message's influence on the ciphertext extends the single faulted operation. To bypass this problem, it is necessary that the encryptions with and without fault injection operate on pairs of equal messages. The two ciphertexts of the pair will be different except for the rare case where the normal value (without fault) of the faulted instruction's output is precisely equal to the value $a$ imposed by the fault. The obtained collision, or more precisely the identity of the ciphertexts, makes evident that the induced fault had no effect. That is, the considered data 
a priori possessed the value which the fault was going to impose. The observation and exploitation of such coincidences is so called Ineffective Fault Analysis (IFA) [5,6]. This chosen message technique needs much more fault injections than CFA but allows to target operations deeper in the algorithm. Note that in some sense IFA may also be seen as a particular case of Safe Error Analysis (SEA) techniques [8,11,12].

Our third attack is based on the classical Differential Fault Analysis (DFA) first introduced by Biham and Shamir [4] who applied it to the DES block cipher. DFA is a general known plaintext technique which exploits the difference between a normal and a faulty ciphertext stemming from encryptions of the same message. As for the attack described in [4], we analyze a fault occurring near the end of the algorithm and assume the general random fault model where the fault modifies the processed data in a random way. The adversary does not need to know a priori the random value the fault imposed on the data, and, as will be shown later, the assumption of cycle accurate fault induction can be relaxed to a certain extend. Furthermore, our attack needs only very few fault injections, which makes it both practical and very efficient.

\section{Collision Fault Analysis of IDEA}

Assuming a fault model where an adversary is able to set the output of the $\boxplus$ operation to a fixed and known value $a$, CFA allows to recover the subkeys $Z_{2}^{1}$ and $Z_{3}^{1}$ at the cost of only two fault injections and on average $2 \times 2^{15}$ encryptions. Given a faulty ciphertext where the fault occurred on the $X_{2}^{1} \boxplus Z_{2}^{1}$ operation, the adversary just has to exhaustively search for that value of $X_{2}^{1}$ which leads to the same output. Then she knows that $X_{2}^{1} \boxplus Z_{2}^{1}=Y_{2}^{1}=a$ and infers the subkey value $Z_{2}^{1}=a \boxminus X_{2}^{1}$ (where $\boxminus$ denotes subtraction modulo $2^{16}$ ). Faulting on the $X_{3}^{1} \boxplus Z_{3}^{1}$ operation will reveal $Z_{3}^{1}$ in the same manner.

Following the same principle, it is possible to retrieve $Z_{1}^{1}$ and $Z_{4}^{1}$ assuming that the same fault model also holds for the $\odot$ operation. We note that for a general purpose microprocessor it is doubtable whether it is possible to force the output of the multiplication to a fixed and known value in the same manner as for the addition operation. While the addition is probably implemented using a single instruction (at least on 16 or 32 bit systems) which can be faulted relatively easy, the multiplication is necessarily composed of several instructions. However, one could assume that the fault model allows to simply set the output of the last instruction in the sequence to $a$. 
In the best case, an adversary might thus recover 64 key bits using this strategy. We do not see any opportunity to recover more key bits applying CFA $^{1}$. Suppose for a moment that the adversary was able to retrieve $Z_{i}^{1}(i=1, \ldots, 4)$. In this case, it is possible to determine the inputs $p$ and $q$ of the first round's multiplication-addition $(M A)$ layer. However, a fault injection on any operation of the $M A$ layer does not allow to find another message that provokes a collision in the ciphertext. For that, it would be necessary that the part of the message which the adversary varies in order to find the collision (for example $X_{1}^{1}$ or $X_{3}^{1}$ if the fault is induced on $p \odot Z_{5}^{1}$ ) only influences the result of the faulted operation (here $p \odot Z_{5}^{1}$ ). Clearly, this condition is not met here because the message entering the first round (and thus the $M A$ layer) is involved again at the end of the same round when it is XOR-ed with the output of the $M A$ layer. Therefore, it is impossible to find a collision that allows to derive $Z_{5}^{1}$ and $Z_{6}^{1}$ by means of CFA.

In the next section, we discuss how this attack can be extended in order to extract supplementary key material using a more elaborate method.

\section{Ineffective Fault Analysis of IDEA}

We consider the case where it is possible to set the output of the addition and multiplication operations to a known and constant value $a$. The 64 bits of the subkeys $Z_{1}^{1}, \ldots, Z_{4}^{1}$ may have been previously determined by CFA such that the adversary is capable to calculate and control $Y_{1}^{1}, \ldots, Y_{4}^{1}$ as well as $p$ and $q$ entering the first round's $M A$ layer. Successively encrypting pairs of messages (normal/faulted) where the fault is induced on the multiplication $\left(p \odot Z_{5}^{1}\right)$, it is possible to determine the value of $\left(p \odot Z_{5}^{1}\right)$ as soon as an identity ${ }^{2}$ of ciphertexts is observed. Such a winning pair allows to reveal the value of $Z_{5}^{1}$ and is obtained after encrypting $2^{15}$ pairs on average since the adversary is able to control the multiplication's input $p$.

Knowing $Z_{5}^{1}$, it is now possible to control the input $s$ of the operation $\left(s \odot Z_{6}^{1}\right)$, and to determine $Z_{6}^{1}$ in the same manner. 96 key bits are hence known and the remaining 32 bits can be found by means of exhaustive search. The attack requires four faults for the CFA phase and on average $2 \times 2^{15}$ additional faults for the IFA phase.

\footnotetext{
${ }^{1}$ Except, however, for an adversary able to also decrypt chosen ciphertexts. Such an adversary can retrieve both $Z_{1}^{1}$ to $Z_{4}^{1}$ and $Z_{1}^{9}$ to $Z_{4}^{9}$ which amounts to 86 out of the 128 key bits.

${ }^{2}$ We consciously avoid the term collision since the algorithm's inputs are the same.
} 


\section{Differential Fault Analysis of IDEA}

Our attack applies to the last 1.5 rounds of IDEA and recovers 93 out of 128 key bits. The remaining 35 key bits may be recovered by exhaustive search. Most symbols used in the description refer to the notation introduced in Section 2. However, since the attack involves the output transformation, we depict the construction considered in Figure 3. Note that the figure takes into account the double permutation of the branches two and three at the end of round eight and the beginning of round nine, leading to a permutation of $X_{3}^{9}$ and $X_{2}^{9}$. The attack is split into three successive phases.

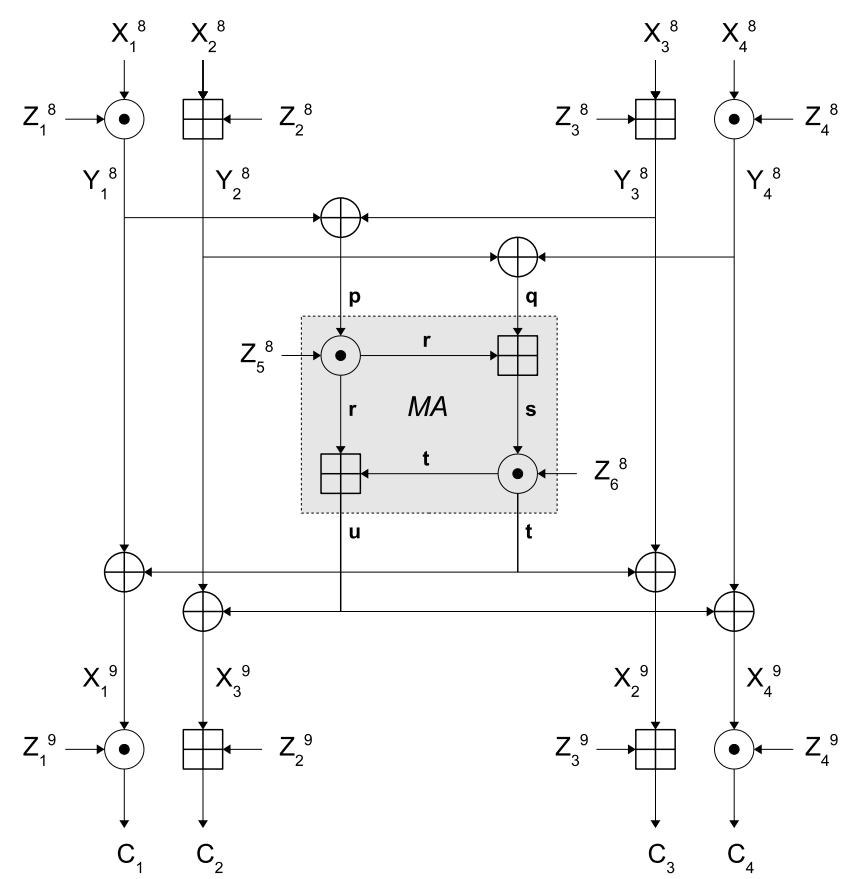

Fig. 3. Last 1.5 rounds of IDEA

\subsection{Phase 1: finding the subkeys of the output transformation}

The first phase of the attack aims at recovering the subkeys $Z_{1}^{9}, \ldots, Z_{4}^{9}$ which are used in the output transformation (cf. Figure 3). Taking into account that our attack does not allow to recover the most significant bits of $Z_{2}^{9}$ and $Z_{3}^{9}$, we thus reveal 62 key bits. 
The fault injection targets at the last round's $M A$ layer, highlighted by a gray box in Figure 3. A fault may be induced on either $p, q, r$, or $s$ whereas the approach is identical in either case. Note that any modification of one of these values provokes a priori a XOR-difference $\Delta_{u}$ on $u$ and $\Delta_{t}$ on $t$. These alter the reference ciphertext $\left(C_{1}, C_{2}, C_{3}, C_{4}\right)$ into the faulty ciphertext $\left(C_{1}^{*}, C_{2}^{*}, C_{3}^{*}, C_{4}^{*}\right)$. One can observe that

$$
X_{1}^{9} \oplus X_{1}^{9 *}=X_{2}^{9} \oplus X_{2}^{9 *}=\Delta_{t}
$$

holds, since $Y_{1}^{8}=Y_{1}^{8 *}$ and $Y_{3}^{8}=Y_{3}^{8 *}$, i.e. $Y_{1}^{8}$ and $Y_{3}^{8}$ are not affected by the fault. Similarly, also

$$
X_{3}^{9} \oplus X_{3}^{9 *}=X_{4}^{9} \oplus X_{4}^{9 *}=\Delta_{u}
$$

holds, because $Y_{2}^{8}$ and $Y_{4}^{8}$ are not affected by the fault.

The relation in Equation 1 in conjunction with a pair of reference and faulty ciphertexts allows to infer a relation between $Z_{1}^{9}$ and $Z_{3}^{9}$ which can be exploited to restrict a list of possible candidates for the value of the couple $\left(Z_{1}^{9}, Z_{3}^{9}\right)$. Given the pair $\left(C_{1}, C_{1}^{*}\right)$ an adversary is able to derive

$$
\Delta_{t}=\left(C_{1} \odot\left(Z_{1}^{9}\right)^{-1}\right) \oplus\left(C_{1}^{*} \odot\left(Z_{1}^{9}\right)^{-1}\right)
$$

for any arbitrary guess on the value of $Z_{1}^{9}$. Using the pair $\left(C_{3}, C_{3}^{*}\right)$ she can generate a list of candidate values for $Z_{3}^{9}$ which are compatible with this $\Delta_{t}$. More precisely, the candidate values for $Z_{3}^{9}$ need to satisfy

$$
\left(C_{3} \boxminus Z_{3}^{9}\right) \oplus\left(C_{3}^{*} \boxminus Z_{3}^{9}\right)=\Delta_{t} .
$$

This procedure leads to a list of tuples $\left(Z_{1}^{9}, Z_{3}^{9}\right)$ which are compatible under the observed fault injection.

Applying the above procedure to multiple pairs of reference and faulty ciphertexts obtained from independently performed fault injections, we can progressively further restrict this list of possible candidates for the couple more and more since the correct candidate necessarily appears in the intersection of the lists deduced from each pair of ciphertexts. With only a few faults (cf. Section 7) it is hence possible to definitely identify the value of $Z_{1}^{9}$ and to determine $Z_{3}^{9}$ except for its most significant bit. This bit can not be identified because if $Z_{3}^{9}$ verifies Equation 3 then this also holds for $Z_{3}^{9} \oplus 0 x 8000$. This simply comes from the fact that adding (or subtracting) $0 x 8000$ modulo $2^{16}$ or XOR-ing with $0 x 8000$ are just equivalent operations. 
The same approach can be mounted to derive the values of $Z_{2}^{9}$ and $Z_{4}^{9}$ using the relation in Equation 2. Given the pair $\left(C_{4}, C_{4}^{*}\right)$ the adversary derives

$$
\Delta_{u}=\left(C_{4} \odot\left(Z_{4}^{9}\right)^{-1}\right) \oplus\left(C_{4}^{*} \odot\left(Z_{4}^{9}\right)^{-1}\right)
$$

for any arbitrary guess on $Z_{4}^{9}$. Then she uses

$$
\left(C_{2} \boxminus Z_{2}^{9}\right) \oplus\left(C_{2}^{*} \boxminus Z_{2}^{9}\right)=\Delta_{u}
$$

to build the list of candidates for the tuple $\left(Z_{2}^{9}, Z_{4}^{9}\right)$ compatible with the observed fault. For the same reason as mentioned above, building the intersection of several lists deduced from multiple pairs of ciphertexts allows to definitely identify $Z_{4}^{9}$ and to determine $Z_{2}^{9}$ except for its most significant bit.

Note that each fault injection observed during this phase can be used independently to generate a list of candidates for the tuple $\left(Z_{1}^{9}, Z_{3}^{9}\right)$ as well as for the tuple $\left(Z_{2}^{9}, Z_{4}^{9}\right)$. This fact makes phase one very efficient in terms of recovered bits per fault injection. At the end of this phase we have derived four candidates for the value of $\left(Z_{1}^{9}, Z_{2}^{9}, Z_{3}^{9}, Z_{4}^{9}\right)$ which corresponds to knowing the key bits 22 to 85 except for bits 38 and 54 .

\subsection{Phase 2: finding the subkey $Z_{6}^{8}$}

The second phase of the attack aims at recovering the 16 key bits 109-124 which are used as subkey $Z_{6}^{8}$ in the penultimate round (cf. Table 1).

A fault may be induced on $Y_{2}^{8}$, or any other preceding value as long as $Y_{1}^{8}, Y_{3}^{8}$, and $Y_{4}^{8}$ remain unmodified, leading to the XOR-difference $\Delta_{q}$ on $q$ (as well as the XOR-differences $\Delta_{t}$ and $\Delta_{u}$ ). Note that the attack also works equivalently when faulting on $Y_{4}^{8}$ or related preceding values as stated above for $Y_{2}^{8}$. Given a pair of reference and faulty ciphertexts and the subkey candidates revealed in the previous phase, an adversary can compute $\Delta_{t}$ and $\Delta_{u}$ as defined in Equations $(3,4)$. Note that the existence of two candidates for $Z_{2}^{9}$ does not matter here, since both candidates lead to the same $\Delta_{u}$ and $\Delta_{t}$. Knowing these values allows to calculate $\Delta_{q}$ being the XOR-difference $\left(Y_{2}^{8} \oplus Y_{2}^{8 *}\right)$ introduced by the fault. The potential to calculate $\Delta_{q}$ is interesting in two ways. It will not only enable the success of this phase but can also be useful in the more general setting of device characterization as it allows to precisely determine the effect of the fault.

The adversary generates the list $T R$ of all tuples $(t, r)$ that are compatible with the observed $\Delta_{t}$ and $\Delta_{u}$. A compatible tuple $(t, r)$ verifies

$$
(r \boxplus t) \oplus\left(r \boxplus\left(t \oplus \Delta_{t}\right)\right)=\Delta_{u} .
$$


For any arbitrary guess on $Z_{6}^{8}$ the adversary checks whether there exists at least one tuple $(t, r)$, such that

$$
(s \boxminus r) \oplus\left(s^{*} \boxminus r\right)=\Delta_{q}
$$

with $s=t \odot\left(Z_{6}^{8}\right)^{-1}$ and $s^{*}=\left(t \oplus \Delta_{t}\right) \odot\left(Z_{6}^{8}\right)^{-1}$. If no such $(t, r)$ exists, the guessed $Z_{6}^{8}$ is discarded from the list of subkey candidates.

Applying the above procedure to multiple pairs of reference and faulty ciphertexts obtained from independently performed fault injections, the list of remaining candidates for $Z_{6}^{8}$ reduces rapidly when building the intersection. Finally, the adversary reveals the correct subkey value.

\subsection{Phase 3: finding the subkey $Z_{5}^{8}$}

The third phase of the attack aims at recovering the 16 key bits $93-108$ which are used as subkey $Z_{5}^{8}$ in the penultimate round (cf. Table 1).

A fault may be induced on $Y_{1}^{8}$ leading to the XOR-difference $\Delta_{p}$ on the input $p$ to the $M A$ layer (as well as the XOR-differences $\Delta_{t}$ and $\Delta_{u}$ on its outputs). As for phase 2 , any timing location of the fault which results in a modification of either $Y_{1}^{8}$ or $Y_{3}^{8}$ would be equivalently exploitable. Given a pair of reference and faulty ciphertexts and the previously derived subkeys $Z_{1}^{9}, \ldots, Z_{4}^{9}$, and $Z_{6}^{9}$ an adversary can compute $\Delta_{t}, \Delta_{u}$, and $\Delta_{p}$ as described for the second phase. We exhaustively consider all possible values for the tuple $(t, r)$. For each of them, we compute $t^{*}=t \oplus \Delta_{t}$, $r^{*}=\left((r \boxplus t) \oplus \Delta_{u}\right) \boxminus t^{*}, s=t \odot\left(Z_{6}^{8}\right)^{-1}$, and $s^{*}=t^{*} \odot\left(Z_{6}^{8}\right)^{-1}$. We discard a candidate for $(t, r)$ if it does not verify $\Delta_{q}=0$, i.e. a valid candidate for the tuple must satisfy

$$
s \boxminus r=s^{*} \boxminus r^{*} .
$$

For all values $Z_{5}^{8}$ we check whether at least one of the remaining candidates for $(t, r)$ satisfies

$$
\left(r \odot\left(Z_{5}^{8}\right)^{-1}\right) \oplus\left(r^{*} \odot\left(Z_{5}^{8}\right)^{-1}\right)=\Delta_{p} .
$$

If this is not the case, we discard this candidate for $Z_{5}^{8}$.

As before, applying the above procedure to multiple pairs of reference and faulty ciphertexts obtained from independently performed fault injections progressively eliminates wrong candidate values.

While simulating this phase of the attack, we observed that a very small number of faults (approximately five) always suffices to reduce the list of possible values for $Z_{5}^{8}$ to only two candidates. Contrarily to the 
phenomenon regarding the most significant bits of $Z_{2}^{9}$ and $Z_{3}^{9}$ pointed out in the first phase, the ambiguity between these two remaining values is not absolute. In our experiments we observed that it might be removed, but it seems to be difficult and often happens only after several tens of faults ${ }^{3}$.

To simplify our analysis, we hence consider the third phase a success a soon as at most two candidates for $Z_{5}^{8}$ remain. Consequently, we stipulate that this phase reveals only 15 additional key bits.

\subsection{Summary}

Put together, the three phases of our attack allow to recover 93 key bits. The following section presents simulation results which validate our approach and give a precise estimation about how much fault injections each phase requires.

Remark 1. A trick allows to reduce the required number of faults. Indeed, one may notice that faults injected on $Y_{2}^{8}$ which are used for the second phase may also be partly useful for the first phase. This is because any change of $Y_{2}^{8}$ leads to a differential $\Delta_{t}$ which may be exploitable in phase 1 as neither $Y_{1}^{8}$ nor $Y_{3}^{8}$ is modified. In the same way, a fault modifying $Y_{1}^{8}$ in phase 3 is usable in phase 1 by exploiting the differential $\Delta_{u}$. As a result, injecting $n$ faults for each one of phase 2 and phase 3 allows to save $n$ faults for the first phase.

\section{Simulation Results}

In this section we evaluate the efficiency of all three phases of our attack by means of simulation. The results we present here are based on about 10000 simulations for each of the two first phases and on about 3000 simulations for the third phase, which requires a longer calculation time.

We use two metrics to judge the efficiency of each phase of the attack. The first is the mean residual entropy of the subkey(s) considered after having exploited $m$ faults. It is defined as

$$
h_{m}\left(Z_{i}^{n}\right)=\left\langle\log _{2}\left(\# S_{m}\right)\right\rangle
$$

\footnotetext{
${ }^{3}$ Without having found a satisfactory explication for this phenomenon, we have however observed that for each value of $Z_{5}^{8}$ there exists a companion $\widetilde{Z_{5}^{8}}$ having the property that the values $\Delta_{r}=\left(p \odot Z_{5}^{8}\right) \oplus\left(p^{*} \odot Z_{5}^{8}\right)$ and $\widetilde{\Delta_{r}}=\left(p \odot \widetilde{Z_{5}^{8}}\right) \oplus\left(p^{*} \odot \widetilde{Z_{5}^{8}}\right)$, if $p$ and $p^{*}$ are chosen randomly, are astonishingly close in terms of Hamming Distance and often equal. This observation is worthy of a deeper analysis and it would be interesting to study if it could open new paths for the cryptanalysis of IDEA.
} 
where $Z_{i}^{n}$ denotes the subkey(s) concerned, $\langle\cdot\rangle$ is the expectation operator which we evaluate by means of the empirical mean, and $\# S_{m}$ denotes the size of the set of remaining candidates for $Z_{i}^{n}$ after $m$ faults. An attack aims at reducing this uncertainty.

The second is a kind of success probability, defined as the probability

$$
p\left(\# S_{m} \leqslant d\right)
$$

that the number of remaining candidates is less or equal than $d$ after having exploited $m$ faults. An attack aims at increasing this probability.

Note that both metrics are sound since the filtering processes can not exclude the correct candidate(s) from the list of possible values. Hence, both $h_{m}\left(Z_{i}^{n}\right)=0$ and $\# S_{m} \leqslant 1$ are equivalent to exactly identifying the correct subkey value.

\subsection{Phase 1}

Here the fault may occur on either $p, q, r$, or $s$, i.e. the fault location is flexible to a certain extend. We present detailed results only for the $Z_{1}^{9}$ and $Z_{3}^{9}$ branch since the results for $Z_{2}^{9}$ and $Z_{4}^{9}$ are virtually identical. Further, we also present results for the entire set $Z_{1-4}^{9}$ as this is of most interest. Figure 4 shows the mean residual entropies $h_{m}\left(Z_{1}^{9}\right), h_{m}\left(Z_{3}^{9}\right)$, $h_{m}\left(\left(Z_{1}^{9}, Z_{3}^{9}\right)\right)$, and $h_{m}\left(Z_{1-4}^{9}\right)$ after faulting $m=1,2, \ldots, 10$ times, where each time a random message has been used. One can observe that, as previously argued, revealing the value of $Z_{3}^{9}$ is harder than finding the value of $Z_{1}^{9}$. Therefore, the entropy of the tuple $\left(Z_{1}^{9}, Z_{3}^{9}\right)$ is dominated by the entropy of $Z_{3}^{9}$. Further, $h\left(Z_{1-4}^{9}\right)$ is exactly the double of $h\left(Z_{1}^{9}, Z_{3}^{9}\right)$ which is obviously due to the mutual independence of the two branches. Note that a small number of faults suffices to rapidly approach the residual entropy's intrinsic limit of two bits (the two most significant bits of $Z_{2}^{9}$ and $Z_{3}^{9}$ ). After only five faults, the initial entropy of 64 bits is reduced to 2.38 .

Figure 5 depicts the success probabilities $p\left(\# S_{m} \leqslant 1\right)$ for $Z_{1}^{9}, p\left(\# S_{m} \leqslant\right.$ $2)$ for $Z_{3}^{9}, p\left(\# S_{m} \leqslant 2\right)$ for $\left(Z_{1}^{9}, Z_{3}^{9}\right)$, and $p\left(\# S_{m} \leqslant 4\right)$ for $\left(Z_{1-4}^{9}\right)$ after faulting $m=1,2, \ldots, 10$ times. After only five faults these probabilities are $97 \%, 83 \%, 83 \%$, and $70 \%$ respectively. Still exploiting only five faults, the probability $p\left(\# S_{m} \leqslant 8\right)$ for $\left.Z_{1-4}^{9}\right)$ is $93 \%$.

Another interesting question is whether one of the fault locations, i.e. $p, q, r$, and $s$, is particularly efficient and hence preferable for an attack. Our simulations show that this is not the case. The results for fixed fault 


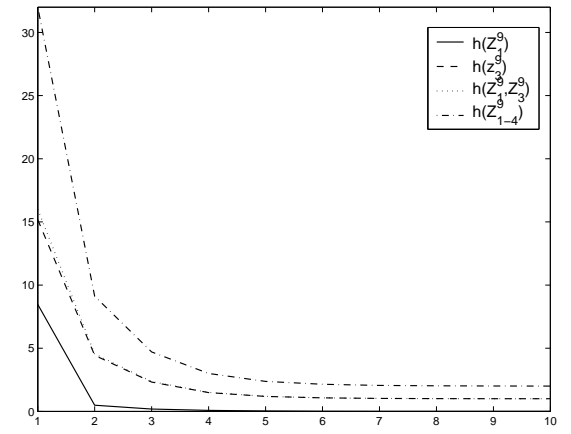

Fig. 4. Mean residual entropy against number of fault injections

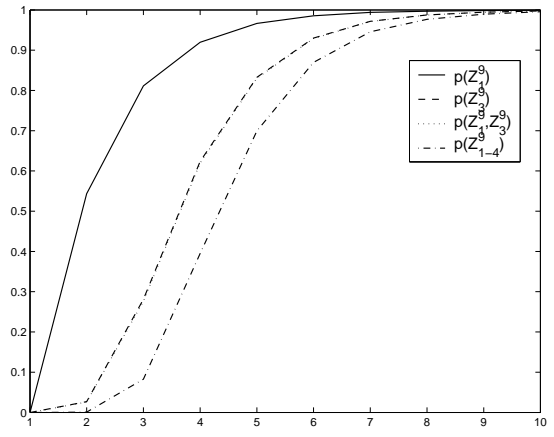

Fig. 5. Probability of $\# S_{m} \leqslant d$ for $Z_{i}^{9}$ against number of fault injections

locations differ only at a negligible order of magnitude and can clearly be considered noise that will vanish for a huge number of simulations.

We also tested whether using a fixed message for the consecutive fault injections during one simulation could be advantageous. We observed that this is not the case. For 2000 simulations with a fixed message and 2000 simulations with a randomly chosen message we obtain, for the set of subkeys $Z_{1-4}^{9}$, an entropy of 6.44 bits in the case of a fixed message and only 4.72 bits in the case of a randomly chosen message after exploiting three faults.

\subsection{Phase 2}

For the second phase, just like for the first one, a small number of fault injections suffices to rapidly reduce the number of remaining candidates for subkey $Z_{6}^{8}$. Figure 6 shows the mean residual entropy $h_{m}\left(Z_{6}^{8}\right)$ after faulting $m=1,2, \ldots, 10$ times, where each time a random message has been used. One can observe that after only five faults there remain less than four candidates in the average case. After eight faults, the mean residual entropy is no more than 0.41 bits and the correct subkey is unambiguously identified in $62 \%$ of the cases.

\subsection{Phase 3}

The third phase of our attack is particularly efficient. As mentioned in the description of the attack, we consider this phase a success as soon as the number of remaining candidates for subkey $Z_{5}^{8}$ is at most two. As can be seen in Figure 9 only four faults suffice to obtain an even better result 


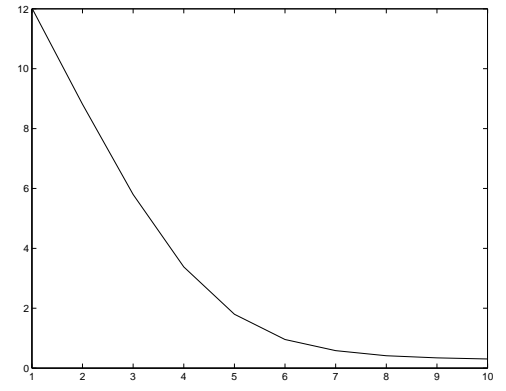

Fig. 6. Mean residual entropy of $Z_{6}^{8}$ against number of fault injections

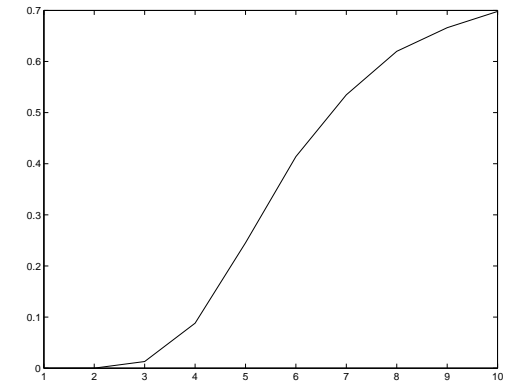

Fig. 7. Probability of $\# S_{m} \leqslant 1$ for $Z_{6}^{8}$ against number of fault injections

in the average case, i.e. the mean residual entropy is less than one bit. Exploiting only three faults, the probability that the number of remaining candidates for $Z_{5}^{8}$ is at most two is greater than $74 \%$.

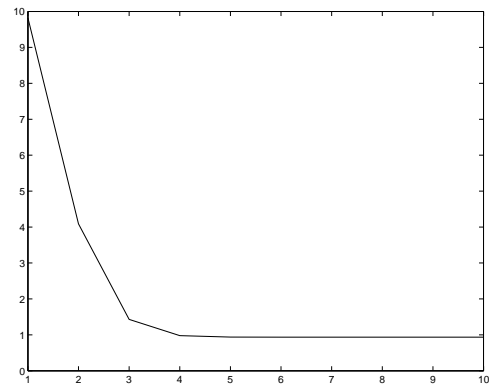

Fig. 8. Mean residual entropy of $Z_{5}^{8}$ against number of fault injections

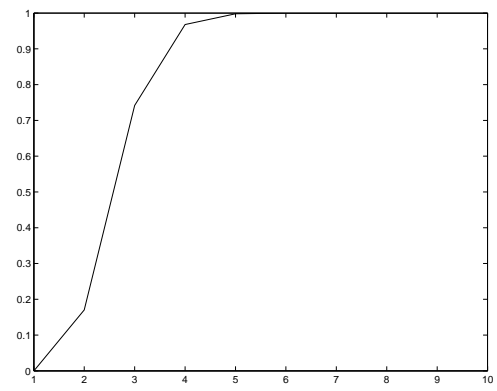

Fig. 9. Probability of $\# S_{m}<=2$ for $Z_{5}^{8}$ against number of fault injections

\section{Conclusion}

In this work we have studied the applicability and efficiency of several fault attacks against the IDEA block cipher. We conclude that CFA can only infer 64 key bits which renders the necessary exhaustive key search for the remaining 64 key bits practically infeasible. On the other hand, IFA allows to retrieve the entire key in the same restrictive fault model but requires a large number of fault injections. For these practical reasons, the third attack presented is of particular interest. Our DFA which is to 
the best of our knowledge the first Differential Fault Analysis of IDEA requires no hypothesis on the effect of the fault and efficiently extracts 93 out of 128 key bits exploiting about only 10 faults. Further, we can also relax the assumption of cycle accurate fault injection to a certain extend which makes the attack even more practical.

IDEA's design makes it by default more resistant to CFA/IFA than other ciphers (e.g. based on SP networks like AES). However, as demonstrated in this paper, implementations of IDEA clearly need to be protected against fault attacks.

\section{Acknowledgments}

The work described in this document has been partly financially supported by the European Commission through the IST Program under Contract IST-2002-507932 ECRYPT.

It was also supported in part by the IAP Programme P6/26 BCRYPT of the Belgian State (Belgian Science Policy), by FWO projects EMA G.0475.05 and BBC G.0300.07, by the European Commission FP6 MIRG project SESOC, number MIRG-CT-2004-516568, and by the K.U.LeuvenBOF (OT/06/40).

The information in this document reflects only the author's views, is provided as is and no guarantee or warranty is given that the information is fit for any particular purpose. The user thereof uses the information at its sole risk and liability. 


\section{References}

1. Frédéric Amiel, Christophe Clavier and Michael Tunstall. Fault Analysis of DPAResistant Algorithms. In Luca Breveglieri, Israel Koren, David Naccache and JeanPierre Seifert, editors, Fault Diagnosis and Tolerance in Cryptography - FDTC'06, volume 4236 of Lecture Notes in Computer Science, pp. 223-236. Springer-Verlag, 2006.

2. Eli Biham, Orr Dunkelman and Nathan Keller. A New Attack on 6-Round IDEA. In Alex Biryukov, editor, Fast Software Encryption - FSE'07, volume 4593 of Lecture Notes in Computer Science, pp. 211-224. Springer-Verlag, 2007.

3. Eli Biham and Adi Shamir. Differential Cryptanalysis of DES-Like Cryptosystems. In Alfred Menezes and Scott A. Vanstone, editors, Advances in Cryptology - CRYPTO '90, volume 537 of Lecture Notes in Computer Science, pp. 2-21. Springer-Verlag, 1991.

4. Eli Biham and Adi Shamir. Differential Fault Analysis of Secret Key Cryptosystems. In Burton S. Kaliski, Jr, editor, Advances in Cryptology - CRYPTO '97, volume 1294 of Lecture Notes in Computer Science, pp. 513-525. Springer-Verlag, 1997.

5. Johannes Blömer and Jean-Pierre Seifert. Fault Based Cryptanalysis of the Advanced Encryption Standard (AES). In Rebecca N. Wright, editor, Financial Cryptography - FC'03, volume 2742 of Lecture Notes in Computer Science, pp. 162-181. Springer-Verlag, 2003.

6. Christophe Clavier. Secret External Encodings Do not Prevent Transient Fault Analysis. In Pascal Paillier and Ingrid Verbauwhede, editors, Cryptographic Hardware and Embedded Systems - CHES'07, volume 4727 of Lecture Notes in Computer Science, pp. 181-194. Springer-Verlag, 2007.

7. Ludger Hemme. A Differential Fault Attack Against Early Rounds of (Triple-)DES. In Marc Joye and Jean-Jacques Quisquater, editors, Cryptographic Hardware and Embedded Systems - CHES '04, volume 3156 of Lecture Notes in Computer Science, pp. 254-267. Springer-Verlag, 2004.

8. Marc Joye, Jean-Jacques Quisquater, Sung-Ming Yen and Moti Yung. Observability Analysis - Detecting When Improved Cryptosystems Fail. In Bart Preneel, editor, Topics in Cryptology - CT-RSA '02, volume 2271 of Lecture Notes in Computer Science, pp. 17-29. Springer-Verlag, 2002.

9. Xuejia Lai and James L. Massey. Markov Ciphers and Differentail Cryptanalysis. In Donald W. Davies, editor, Advances in Cryptology - EUROCRYPT'91, volume 547 of Lecture Notes in Computer Science, pp. 17-38. Springer-Verlag, 1991.

10. Kerstin Lemke, Kai Schramm and Christof Paar. DPA on n-Bit Sized Boolean and Arithmetic Operations and Its Application to IDEA, RC6, and the HMACConstruction. In Marc Joye and Jean-Jacques Quisquater, editors, Cryptographic Hardware and Embedded Systems - CHES'04, volume 3156 of Lecture Notes in Computer Science, pp. 205-219. Springer-Verlag, 2004.

11. Sung-Ming Yen and Marc Joye. Checking Before Output May Not Be Enough Against Fault-Based Cryptanalysis. IEEE Transactions on Computers, 49(9):967$970,2000$.

12. Sung-Ming Yen, Seungjoo Kim, Seongan Lim and Sang-Jae Moon. A Countermeasure against One Physical Cryptanalysis May Benefit Another Attack. In Kwangjo Kim, editor, Information Security and Cryptology - ICISC'01, volume 2288 of Lecture Notes in Computer Science, pp. 414-427. Springer-Verlag, 2002.

13. http://www.mediacrypt.com/, September 17, 2007 